\title{
COMBINATORIAL SOLUTION OF CERTAIN SYSTEMS OF LINEAR EQUATIONS INVOLVING $(0,1)$ MATRICES
}

\author{
W. E. LONGSTAFF \\ (Received 23 February 1976; revised 16 June 1976) \\ Communicated by W. D. Wallis
}

\begin{abstract}
If $m$ and $n$ are natural numbers satisfying $1 \leqq m<n$ let $\left\langle\begin{array}{c}n \\ m\end{array}\right\rangle$ denote the least integer $k$ such that the statement:

'Every $(0,1)$ matrix with $n$ columns, with constant row-sum $m$, and with at least $k$ distinct rows, has rank $n$ '

is true. Then $\left\langle\begin{array}{c}n \\ m\end{array}\right\rangle=\left(\begin{array}{c}n-1 \\ m\end{array}\right)+1$ for $m \geqq 2, n \geqq m^{2}+2$. Further, $\left\langle\begin{array}{c}n \\ m\end{array}\right\rangle=\left\langle\begin{array}{c}n \\ n-m\end{array}\right\rangle$ for $1 \leqq m<n$.
\end{abstract}

\section{Introduction}

Let $m$ and $n$ be natural numbers satisfying $1 \leqq m<n$. With every non-empty family $\mathscr{F}$ of distinct $m$-element subsets of $\{1,2,3, \cdots, n\}$ can be associated a homgeneous system of equations in the real unknowns $x_{1}, x_{2}, x_{3}, \cdots, x_{n}$ :

$$
\sum_{i \in A} x_{i}=0 \quad(A \in \mathscr{F})
$$

The coefficient matrix of this system is a $(0,1)$ matrix with $n$ columns, $|\mathscr{F}|$ distinct rows and constant row-sum $m$. Which such matrices have rank $n$ ? Equivalently: Which such systems have $x_{1}=x_{2}=x_{3}=\cdots=x_{n}=0$ as their only solution? Let $\left\langle\begin{array}{c}n \\ m\end{array}\right\rangle$ denote the least integer $k$ such that the statement:

'For every non-empty family $\mathscr{F}$ of $m$-element subsets of $\{1,2,3, \cdots, n\}$ with at least $k$ distinct elements the system $\sum_{i \in A} x_{i}=0(A \in \mathscr{F})$ has $x_{1}=x_{2}=x_{3}=\cdots=x_{n}=0$ as its only solution' 
is true. Notice that $k=\left(\begin{array}{c}n \\ m\end{array}\right)$ has this property and that $\left\langle\begin{array}{l}n \\ 1\end{array}\right\rangle=n$ for $n \geqq 2$. For each $m \geqq 1,\left\langle\begin{array}{c}n \\ m\end{array}\right\rangle$ is evaluated for all but a finite number of $n$. In particular, it is shown that $\left\langle\begin{array}{c}n \\ m\end{array}\right\rangle=\left(\begin{array}{c}n-1 \\ m\end{array}\right)+1$ for $m \geqq 2, n \geqq m^{2}+2$. The proof uses certain combinatorial inequalities. Also, it is shown that $\left\langle\begin{array}{c}n \\ m\end{array}\right\rangle=$ $\left\langle\begin{array}{c}n \\ n-m\end{array}\right\rangle$ for $1 \leqq m<n$. Applications, concerning uniqueness of solution, are made to more general real systems of linear equations.

\section{Main theorem}

Let $1 \leqq m<n$. The family $\mathscr{G}$, consisting of those $m$-element subsets of $\{1,2,3, \cdots, n\}$ which do not contain $n$, has $\left(\begin{array}{c}n-1 \\ m\end{array}\right)$ elements and the system

$$
\sum_{i \in A} x_{i}=0 \quad(A \in \mathscr{G})
$$

does not have $x_{1}=x_{2}=x_{3}=\cdots=x_{n}=0$ as its only solution since $x_{n}$ can be arbitrary. Thus $\left\langle\begin{array}{c}n \\ m\end{array}\right\rangle \geqq\left(\begin{array}{c}n-1 \\ m\end{array}\right)+1$.

THEOREM 2.1. $\left\langle\begin{array}{c}n \\ m\end{array}\right\rangle=\left(\begin{array}{c}n-1 \\ m\end{array}\right)+1$ for $m \geqq 2$ and $n \geqq m^{2}+2$.

Remark. The cancellation law of addition is used in the proof. The proof is divided into five steps and involves several combinatorial inequalities. Establishing the validity of these inequalities, although necessary for the proof, adds nothing to its understanding. The more difficult inequalities are numbered $I_{1}$ to $I_{4}$ and are dealt with in the fifth and final step.

Proof. It suffices to show that if $\mathscr{S}$ is a family of $m$-element subsets of $\{1,2,3, \cdots, n\}$ satisfying $|\mathscr{S}|=\left(\begin{array}{c}n-1 \\ m\end{array}\right)+1$ and $x_{1}, x_{2}, x_{3}, \cdots, x_{n}$ are real numbers satisfying $\sum_{i \in A} x_{i}=\sum_{j \in B} x_{j}(A, B \in \mathscr{S})$ then $x_{1}=x_{2}=x_{3}=\cdots=x_{n}$.

Step 1. It is shown that there is a subset $\mathscr{X}$ of $\{1,2,3, \cdots, n\}$ with $|\mathscr{X}|=2 m-3$ such that $x_{i}=x_{j}$ whenever $i, j \in \mathscr{X}^{c}$ (where $\mathscr{X}^{c}$ denotes the complement of $\mathscr{X}$ ): 
For every $(m-1)$-element subset $S$ of $\{1,2,3, \cdots, n\}$ let $q_{s}$ be the number of elements of $\mathscr{S}$ which contain $S$. Then

$$
\sum_{\text {alls }} q_{s}=m\left\{\left(\begin{array}{c}
n-1 \\
m
\end{array}\right)+1\right\} .
$$

If $q_{s} \geqq n-2 m+3$ we may take $\mathscr{X}$ to be $\mathscr{Y}^{c}$ where $\mathscr{Y}$ is any subset of $\{i: S \cup\{i\} \in \mathscr{P}\}$ of order $n-2 m+3$. Suppose then, that $q_{s} \leqq n-2 m+2$ for every $S$. There exists $S_{1}$ satisfying

$$
\dot{q}_{s_{1}} \geqq \frac{m\left\{\left(\begin{array}{c}
n-1 \\
m
\end{array}\right)+1\right\}}{\left(\begin{array}{c}
n \\
m-1
\end{array}\right)} .
$$

Since

$$
\frac{m\left\{\left(\begin{array}{c}
n-1 \\
m
\end{array}\right)+1\right\}}{\left(\begin{array}{c}
n \\
m-1
\end{array}\right)}>\frac{m\left(\begin{array}{c}
n-1 \\
m
\end{array}\right)}{\left(\begin{array}{c}
n \\
m-1
\end{array}\right)}=\frac{(n-m+1)(n-m)}{n} \geqq n-2 m+1,
$$

it follows that $q_{s_{1}}=n-2 m+2$. Suppose that $k$ distinct $(m-1)$-element subsets $S_{i}$ have been found satisfying $q_{s_{i}}=n-2 m+2$ and that $1 \leqq k \leqq$ $m\left(\begin{array}{c}n-1 \\ m-2\end{array}\right)+m-1$. Since

$$
m\left(\frac{n-1}{m-2}\right)+m-1<\left(\begin{array}{c}
n \\
m-1
\end{array}\right) \quad\left(m \geqq 2, n \geqq m^{2}+2\right),
$$

there is an $(m-1)$-element subset $S_{k+1}$ distinct from $S_{i}(1 \leqq i \leqq k)$ such that

$$
q_{s_{k+1}} \geqq \frac{m\left\{\left(\begin{array}{c}
n-1 \\
m
\end{array}\right)+1\right\}-k(n-2 m+2)}{\left(\begin{array}{c}
n \\
m-1
\end{array}\right)-k} .
$$

It is easy to verify that

$$
\frac{m\left\{\left(\begin{array}{c}
n-1 \\
m
\end{array}\right)+1\right\}-k(n-2 m+2)}{\left(\begin{array}{c}
n \\
m-1
\end{array}\right)-k}>n-2 m+1,
$$

so $q_{s_{k+1}}=n-2 m+2$. It follows that there are $m\left(\begin{array}{c}n-1 \\ m-2\end{array}\right)+m$ distinct $(m-1)$-element subsets $S$ of $\{1,2,3, \cdots, n\}$ satisfying $q_{s}=n-2 m+2$. Let 
$\sigma=m\left(\begin{array}{c}n-1 \\ m-2\end{array}\right)+m$. By using the cancellation law of addition it follows that there are $\sigma$ (possibly not distinct) $(2 m-2)$-element subsets $B_{p}(1 \leqq p \leqq \sigma)$ of $\{1,2,3, \cdots, \boldsymbol{n}\}$ such that

(a) $i, j \in B_{p}^{c}$ (some $p$ ) implies $x_{i}=x_{j}$;

(b) From $\bigcup_{1 \leqq p \leqq \sigma} B_{p}$ can be chosen $\sigma$ distinct $(m-1)$-element subsets.

If $\kappa=\left|\bigcup_{1 \leq p \leqq \sigma} B_{p}\right|$ then, by (b), $\left(\begin{array}{c}\kappa \\ m-1\end{array}\right) \geqq \sigma$. Since

$$
\left(\begin{array}{c}
2 m-2 \\
m-1
\end{array}\right)<m\left(\begin{array}{c}
n-1 \\
m-2
\end{array}\right)+m=\sigma \quad\left(m \geqq 2, n \geqq m^{2}+2\right),
$$

it follows that $\kappa>2 m-2$. Hence $B_{p_{1}} \neq B_{p_{2}}$ for some $p_{1}$ distinct from $p_{2}$. Now $\left|B_{p_{1}} \cap B_{p_{2}}\right| \leqq 2 m-3$ and $\left(B_{p_{1}} \cup B_{p_{2}}\right)^{c}$ is non-empty (since $n \geqq 4 m-3$ ). If $i_{0} \in\left(B_{p_{1}} \cup B_{p_{2}}\right)^{c}$ and $j \in\left(B_{p_{1}} \cap B_{p_{2}}\right)^{c}$ we have $x_{j}=x_{i_{0}}$ by (a). The subset $\mathscr{X}$ can be taken to be any $(2 m-3)$-element subset of $\{1,2,3, \cdots, n\}$ containing $\boldsymbol{B}_{p_{1}} \cap \boldsymbol{B}_{p_{2}}$.

Step 2. It is shown that there is a member $A$ of $\mathscr{S}$ which is disjoint from $\mathscr{X}$ :

This follows from the inequality:

$$
\left(\begin{array}{c}
n-1 \\
m
\end{array}\right) \geqq\left(\begin{array}{c}
n \\
m
\end{array}\right)-\left(\begin{array}{c}
n-2 m+3 \\
m
\end{array}\right) \quad\left(m \geqq 2, n \geqq m^{2}+2\right) .
$$

Step 3. It is shown that, given any $t$ distinct elements of $\{1,2,3, \cdots, n\}$ with $1 \leqq t \leqq 2 m-3$ there is an element of $\mathscr{S}$ which contains one and only one of these $t$ elements:

In all, there are $t\left(\begin{array}{c}n-t \\ m-1\end{array}\right) m$-element subsets of $\{1,2,3, \cdots, n\}$ which contain precisely one of the $t$ given elements. The desired result follows from the inequality:

(I $\left.I_{4}\right)\left(\begin{array}{c}n-1 \\ m\end{array}\right) \geqq\left(\begin{array}{c}n \\ m\end{array}\right)-t\left(\begin{array}{c}n-t \\ m-1\end{array}\right) \quad\left(m \geqq 2, n \geqq m^{2}+2,1 \leqq t \leqq 2 m-3\right)$.

Step 4. Assume that the combinatorial inequalities $I_{1}-I_{4}$ are valid. By the result of Step $1, x_{i}=x_{i}$ whenever $i, j \in \mathscr{Z}^{c}$ and $|\mathscr{X}|=2 m-3$. Let the subset $A$ be as in Step 2. By the result of Step 3, there is an element $B$ of $\mathscr{S}$ which contains precisely one element, $r_{1}$ say, of $\mathscr{X}$. Since $\Sigma_{i \in B} x_{i}=\Sigma_{j \in A} x_{j}$, it follows by cancellation that $x_{r_{1}}=x_{j} \quad\left(j \in \mathscr{X}^{c}\right)$. Thus $x_{i}=x_{j}$ whenever $i, j \in \mathscr{X}^{c} \cup\left\{\boldsymbol{r}_{1}\right\}$. Similarly, by applying the result of Step 3 to the set $\mathscr{Z} \backslash\left\{\boldsymbol{r}_{1}\right\}$ we 
deduce that $x_{r_{2}}=x_{i}\left(j \in \mathscr{Q}^{c} \cup\left\{r_{1}\right\}\right)$ for some $r_{2}$ satisfying $r_{2} \in \mathscr{X} \backslash\left\{r_{1}\right\}$. Thus $x_{i}=x_{j}$ whenever $i, j \in \mathscr{X}^{c} \cup\left\{r_{1}, r_{2}\right\}$. Continuing in this way, we finally obtain the result that $x_{1}=x_{2}=x_{3}=\cdots=x_{n}$.

Step 5. It remains to establish the validity of inequalities $I_{1}-I_{4}$ :

$\left(I_{1}\right): \quad m\left(\begin{array}{c}n-1 \\ m-2\end{array}\right)+m-1<\left(\begin{array}{c}n \\ m-1\end{array}\right) \quad\left(m \geqq 2, n \geqq m^{2}+2\right)$.

This inequality is easily shown to be equivalent to

$$
\left(\begin{array}{c}
n-1 \\
m-2
\end{array}\right)\left[\frac{n}{m(m-1)}-1\right] \geqq 1 \text {. }
$$

The latter is clearly valid for $m=2, n \geqq 6$. If $m \geqq 3, n \geqq m^{2}+2$ we have $\frac{n}{m(m-1)}-1 \geqq 0$ and $\left(\begin{array}{c}n-1 \\ m-2\end{array}\right) \geqq n-1$. Hence,

$$
\left(\begin{array}{c}
n-1 \\
m-2
\end{array}\right)\left[\frac{n}{m(m-1)}-1\right] \geqq(n-1)\left[\frac{n}{m(m-1)}-1\right] \geqq 1 .
$$

$\left(I_{2}\right)$ :

$$
\left(\begin{array}{c}
2 m-2 \\
m-1
\end{array}\right)<m\left(\begin{array}{c}
n-1 \\
m-2
\end{array}\right)+m \quad\left(m \geqq 2, n \geqq m^{2}+2\right) .
$$

Clearly, it suffices to show that

$$
\left(\begin{array}{c}
2 m-2 \\
m-1
\end{array}\right) \leqq\left(\begin{array}{c}
m^{2}+1 \\
m-2
\end{array}\right) \quad(m \geqq 3) .
$$

This is equivalent to showing that

$$
\frac{2^{m-1}}{m-1} \prod_{r=1}^{m-2}(2 m-2 r-1) \leqq \prod_{r=1}^{m-2}\left(m^{2}+2-r\right) \quad(m \geqq 3) .
$$

Since $2^{m-1} /(m-1) \leqq 2^{m-2}$ and $m^{2} \geqq 4 m-7$ for $m \geqq 3$ it follows that

$$
\frac{2^{m-1}}{m-1} \prod_{r=1}^{m-2}(2 m-2 r-1) \leqq \prod_{r=1}^{m-2}(4 m-4 r-2) \leqq \prod_{r=1}^{m-2}\left(m^{2}+2-r\right) .
$$

$\left(I_{3}\right): \quad\left(\begin{array}{c}n-1 \\ m\end{array}\right) \geqq\left(\begin{array}{c}n \\ m\end{array}\right)-\left(\begin{array}{c}n-2 m+3 \\ m\end{array}\right) \quad\left(m \geqq 2, n \geqq m^{2}+2\right)$.

This inequality is easily shown to be equivalent to

$$
\prod_{r=1}^{m-1}\left(\frac{n-3 m+4+r}{n-m+r}\right) \cdot \frac{n-3 m+4}{m} \geqq 1 \quad\left(m \geqq 2, n \geqq m^{2}+2\right) .
$$


Notice that, if $m \geqq 2$ and $n_{1} \geqq n_{2} \geqq m^{2}+2$ we have

$$
\frac{n_{1}-3 m+4+r}{n_{1}-m+r} \geqq \frac{n_{2}-3 m+4+r}{n_{2}-m+r} \quad(1 \leqq r \leqq m-1) .
$$

Therefore, it suffices to show that

$$
\prod_{r=1}^{m-1}\left(\frac{m^{2}-3 m+6+r}{m^{2}-m+2+r}\right) \cdot \frac{m^{2}-3 m+6}{m} \geqq 1 \quad(m \geqq 2) .
$$

If

$$
a_{m}=\prod_{r=1}^{m-1}\left(\frac{m^{2}-3 m+6+r}{m^{2}-m+2+r}\right) \text { for } m \geqq 2
$$

then

$$
a_{m} \geqq\left(\frac{m^{2}-3 m+7}{m^{2}-m+3}\right)^{m-1}=\left(1-\frac{2 b_{m}}{m-1}\right)^{m-1},
$$

where

$$
b_{m}=\frac{(m-1)(m-2)}{m^{2}-m+3} \quad(m \geqq 2) .
$$

Since $b_{m} \leqq 1$ we have $a_{m} \geqq\left(1-\frac{2}{m-1}\right)^{m-1}(m \geqq 2)$. The sequence $c_{m}=$ $\left(1-\frac{2}{m-1}\right)^{m-1}$ is monotone non-decreasing for $m \geqq 2$. Hence $a_{m} \geqq c_{4}=\frac{1}{27}$ for $m \geqq 4$. Thus,

$$
a_{m} \cdot \frac{m^{2}-3 m+6}{m} \geqq \frac{m^{2}-3 m+6}{27 m} \geqq 1 \text { for } m \geqq 30 .
$$

That $a_{m} \cdot\left(m^{2}-3 m+6\right) / m \geqq 1$ is true for $2 \leqq m \leqq 29$ is easily checked directly.

$\left(I_{4}\right):\left(\begin{array}{c}n-1 \\ m\end{array}\right) \geqq\left(\begin{array}{c}n \\ m\end{array}\right)-t\left(\begin{array}{c}n-t \\ m-1\end{array}\right) \quad\left(m \geqq 2, n \geqq m^{2}+2,1 \leqq t \leqq 2 m-3\right)$.

We may suppose $m \geqq 3, n \geqq m^{2}+2,2 \leqq t \leqq 2 m-3$. It is easily shown that this inequality is equivalent to

$$
\prod_{r=0}^{t-2}\left(\frac{n-t+1+r}{n-m-t+2+r}\right) \leqq t \quad\left(m \geqq 3, n \geqq m^{2}+2,2 \leqq t \leqq 2 m-3\right) .
$$


If $n_{1} \geqq n_{2} \geqq m^{2}+2$ and $m \leqq 3,2 \leqq t \leqq 2 m-3$ we have

$$
\frac{n_{1}-t+1+r}{n_{1}-m-t+2+r} \leqq \frac{n_{2}-t+1+r}{n_{2}-m-t+2+r}
$$

Therefore, it suffices to show that

$$
\prod_{r=0}^{t-2}\left(\frac{m^{2}+3-t+r}{m^{2}-m+4-t+r}\right) \leqq t \quad(m \geqq 3,2 \leqq t \leqq 2 m-3) .
$$

Since

$$
\prod_{r=0}^{t-2}\left(\frac{m^{2}+3-t+r}{m^{2}-m+4-t+r}\right) \leqq\left(\frac{m^{2}+3-t}{m^{2}-m+4-t}\right)^{t-1}
$$

it suffices to show that

$$
\frac{m^{2}+3-t}{m^{2}-m+4-t} \leqq t^{\frac{1}{t-1}}
$$

or equivalently, that

$$
t+\frac{m-1}{t^{\frac{1}{1-1}}-1} \leqq m^{2}-m+4 \quad(m \geqq 3,2 \leqq t \leqq 2 m-3) .
$$

Since the function $t \mapsto t^{\frac{1}{t-1}}$ is monotone decreasing it suffices to show that

$$
2 m-3+\frac{m-1}{(2 m-3)^{\frac{1}{2 m-4}}-1} \leqq m^{2}-m+4 \quad(m \geqq 3)
$$

or, that

$$
\left(1+\frac{m-1}{m^{2}-3 m+7}\right)^{2 m-4} \leqq 2 m-3 \quad(m \geqq 3)
$$

Clearly, this is true for $m=3$ or 4 . If $m \geqq 5$ and $x=2 m-4$ we have to show that

$$
1+x \geqq\left(1+\frac{2 x+4}{x^{2}+2 x+20}\right)^{x}
$$

Now

$$
\frac{2 x+4}{x^{2}+2 x+20} \leqq \frac{2}{x} \text { for } x \geqq 6
$$

Also, $1+x \geqq(1+(2 / x))^{x}$ for $x \geqq 6$. The result follows.

This completes the proof of the theorem. 


\section{Extension and application}

The number $\left\langle\begin{array}{c}n \\ m\end{array}\right\rangle$ has been evaluated for $m=1, n \geqq 2$ and for $m \geqq 2$, $n \geqq m^{2}+2$. In fact, $\left\langle\begin{array}{c}n \\ m\end{array}\right\rangle=\left(\begin{array}{c}n-1 \\ m\end{array}\right)+1$ in these cases. The domain of evaluation of $\left\langle\begin{array}{c}n \\ m\end{array}\right\rangle$ can be extended by the following observation:

If $1 \leqq m<n$ we have $\left\langle\begin{array}{c}n \\ m\end{array}\right\rangle=\left\langle\begin{array}{c}n \\ n-m\end{array}\right\rangle$.

For, suppose $\mathscr{S}$ is a family of $m$-element subsets of $\{1,2,3, \cdots, n\}$ with $|\mathscr{S}|=\left\langle\begin{array}{c}n \\ n-m\end{array}\right\rangle$ and take real numbers $x_{1}, x_{2}, x_{3}, \cdots, x_{n}$ satisfying $\Sigma_{i \in A} x_{i}=0$ $(A \in \mathscr{S})$. If $s=\sum_{1 \leqq i \leqq n} x_{i}$ and $y_{i}=x_{i}-s /(n-m)$ we have $\Sigma_{i \in A^{c}} y_{i}=0$ $(A \in \mathscr{S})$. Hence, by definition of $\left\langle\begin{array}{c}n \\ n-m\end{array}\right\rangle, y_{i}=0(1 \leqq i \leqq n)$. Thus $x_{i}=0$ $(1 \leqq i \leqq n)$. This shows that $\left\langle\begin{array}{c}n \\ m\end{array}\right\rangle \leqq\left\langle\begin{array}{c}n \\ n-m\end{array}\right\rangle$. The reverse inequality follows by symmetry.

Thus, for example, $\left\langle\begin{array}{l}6 \\ 4\end{array}\right\rangle=\left\langle\begin{array}{l}6 \\ 2\end{array}\right\rangle=\left\langle\begin{array}{l}5 \\ 2\end{array}\right\rangle+1=11$ and $\left\langle\begin{array}{c}n \\ n-1\end{array}\right\rangle=\left\langle\begin{array}{l}n \\ 1\end{array}\right\rangle=n$ for $n \geqq 2$.

The numbers $\left\langle\begin{array}{c}n \\ m\end{array}\right\rangle$ provide sufficient conditions for the uniqueness of solution of certain homgeneous real linear systems of equations. We now show how these conditions can be applied to a more general class of real linear systems to establish uniqueness of solution.

Let $n, m$ and $k$ be natural numbers satisfying $1 \leqq m<n$ and $k \geqq\left\langle\begin{array}{c}n \\ m\end{array}\right\rangle$. Let $E$ be a real linear system of equations in unknowns $x_{1}, x_{2}, x_{3}, \cdots, x_{n}$ such that

(I) The system reads " $\sum_{j=1}^{n} a_{i j} x_{i}=b(i=1,2,3, \cdots, k)$ "; (That is, $b$ is the same for all equations of the system).

(II) The matrix $\left(a_{i j}\right)$ has precisely $m$ non-zero entries in each row;

(III) The non-zero coefficients of $x_{j}$ are equal;

(IV) No two equations are identical.

THEOREM 3.1. Each system $E$ (as above) has a unique solution, $x_{j}=b / a_{i} m(j=1,2,3, \cdots, n)$ where $a_{i}$ is any non-zero coefficient of $x_{i}$.

Proof. Since $k \geqq\left\langle\begin{array}{c}n \\ m\end{array}\right\rangle \geqq\left(\begin{array}{c}n-1 \\ m\end{array}\right)+1$, every $x_{j}$ has a non-zero coeffi- 
cient $a_{j}$. If we put $y_{j}=a_{i} x_{j}-b / m$ the system becomes $\Sigma_{j \in A} y_{j}=0(A \in \mathscr{P})$, where $\mathscr{S}$ is a family of $m$-element subsets of $\{1,2,3, \cdots, n\}$ with $|\mathscr{S}|=k \geqq$ $\left\langle\begin{array}{c}n \\ m\end{array}\right\rangle$. It follows from the definition of $\left\langle\begin{array}{c}n \\ m\end{array}\right\rangle$ that $y_{j}=0(j=1,2,3, \cdots, n)$. Hence $x_{j}=b / a_{j} m(j=1,2,3, \cdots, n)$ and the theorem is proved.

EXAmple. The system

$$
\left[\begin{array}{llllll}
0 & 0 & 4 & 3 & 2 & 1 \\
0 & 5 & 0 & 3 & 2 & 1 \\
0 & 5 & 4 & 0 & 2 & 1 \\
0 & 5 & 4 & 3 & 2 & 0 \\
6 & 0 & 0 & 3 & 2 & 1 \\
6 & 0 & 4 & 3 & 0 & 1 \\
6 & 0 & 4 & 3 & 2 & 0 \\
6 & 5 & 0 & 3 & 0 & 1 \\
6 & 5 & 0 & 3 & 2 & 0 \\
6 & 5 & 4 & 0 & 0 & 1 \\
6 & 5 & 4 & 3 & 0 & 0
\end{array}\right]\left[\begin{array}{c}
x_{1} \\
x_{2} \\
\cdot \\
\cdot \\
x_{6}
\end{array}\right]=\left[\begin{array}{l}
3 \\
3 \\
\cdot \\
\cdot \\
\cdot \\
\cdot \\
\cdot \\
\cdot \\
\cdot \\
\cdot \\
3
\end{array}\right]
$$

has unique solution $x_{1}=\frac{1}{8}, x_{2}=\frac{3}{20}, x_{3}=\frac{3}{16}, x_{4}=\frac{1}{4}, x_{5}=\frac{3}{8}, x_{6}=\frac{3}{4}$.

Department of Mathematics,

The University of Western Australia,

Nedlands, W.A., 6009,

Australia. 